\title{
Design of a Time Synchronization System based on GPS and IEEE 1588 for Transmission Substations
}

DOI:

10.1109/TPWRD.2016.2600759

\section{Document Version}

Accepted author manuscript

Link to publication record in Manchester Research Explorer

\section{Citation for published version (APA):}

Guo, H., \& Crossley, P. (2017). Design of a Time Synchronization System based on GPS and IEEE 1588 for Transmission Substations. IEEE Transactions on Power Delivery, 32(4), 2091 - 2100.

https://doi.org/10.1109/TPWRD.2016.2600759

\section{Published in:}

IEEE Transactions on Power Delivery

\section{Citing this paper}

Please note that where the full-text provided on Manchester Research Explorer is the Author Accepted Manuscript or Proof version this may differ from the final Published version. If citing, it is advised that you check and use the publisher's definitive version.

\section{General rights}

Copyright and moral rights for the publications made accessible in the Research Explorer are retained by the authors and/or other copyright owners and it is a condition of accessing publications that users recognise and abide by the legal requirements associated with these rights.

\section{Takedown policy}

If you believe that this document breaches copyright please refer to the University of Manchester's Takedown Procedures [http://man.ac.uk/04Y6Bo] or contact uml.scholarlycommunications@manchester.ac.uk providing relevant details, so we can investigate your claim.

\section{OPEN ACCESS}




\title{
Design of a Time Synchronization System based on GPS and IEEE 1588 for Transmission Substations
}

\author{
Hao Guo, Student Member, IEEE, and Peter Crossley, Member, IEEE
}

\begin{abstract}
Accurate timing is often required for the Intelligent Electronic Devices (IEDs) used in transmission substations. A common method of achieving this is direct connection of a device to a local GPS receiver and the use of its 1-PPS synchronizing signal and the IRIG-B coded message. However, concerns about GPS reliability are encouraging the use of timing systems less dependent on the direct use of local GPS receivers. IEEE 1588 protocol is a network based time synchronization technique designed to co-exist with IEC 61850 applications and deliver sub micro-second timing accuracy. Many utilities are now considering the adoption of IEEE 1588, but they need confidence in the reliability of this technology before it can be rolled out to real substations. Hence, comprehensive tests were undertaken on an IEEE 1588 timing system, to help gain insight into the limitations of the system.

This paper presents a procedure to assess the performance of a timing system based on distributed GPS receivers and one based on a mixture of GPS receivers and IEEE 1588 devices. Test results indicate whichever system is selected, high quality devices and systems, with appropriate installation and engineering, are essential to satisfy the stringent $\pm 1 \mu$ s accuracy requirements needed by critical IED applications.
\end{abstract}

Index Terms-Time Synchronization, Global Positioning System, IEEE 1588, Performance Evaluation, Ethernet Networks, IEC 61850, Protection and Control, Power Transmission, Substation Automation

\section{INTRODUCTION}

$\mathrm{S}$ ubstation is a critical part of a power transmission network and the associated Protection and Control (P\&C), or intelligent electronic devices (IEDs), limits the damage caused by a fault, allows switching operations to occur and in extreme situations prevents the power grid from collapsing. In general, an IED measures the local voltage and current signals, and when multiple IEDs are synchronized to a common time reference, they can be used to reflect the system state in real time [1]. This requirement for time synchronization is becoming increasingly important, especially when the network operators require knowledge of the actual system state in real time, or feeder protection is based on differential protection.

Simple local Global Positioning System (GPS) receivers are widely used for accurate timing in the power industry due to their low cost and accessibility. Having received and decoded the time information from satellites, a GPS receiver feeds the time signal to the IEDs, in the format of one-pulse-per-second

This manuscript was submitted for review on $\mathrm{xx} / \mathrm{xx} / \mathrm{xxxx}$. This work was supported in part by the School of Electrical and Electronic Engineering, The University of Manchester and in part by the Engineering and Physical Sciences Research Council Centre for Doctoral Training in Power Networks project.

The authors are with the School of Electrical and Electronic Engineering, The University of Manchester, Manchester, M60 1QD, UK (e-mail: hao.guo-5@postgrad.manchester.ac.uk; peter.crossley@manchester.ac.uk).
(1-PPS) and/or the IRIG-B time code, via dedicated fibre optic cable [2]-[3]. Considering the significant number of IEDs requiring a time source and that a single fibre optic cable may only carry time information to one device, it is inevitable that dozens of distributed GPS receivers are deployed within a power substation [4]. Many utilities and timing experts have raised concern about the reliability of local, inexpensive GPS receivers and a number of protective relay mal-operation caused by incorrect timing data obtained from GPS receivers have been reported [4]-[5]. In addition, GPS jamming [6] also poses a threat to the continuous availability of GPS in a substation and this severely affects the use of synchronized data for automation control, especially when information from multiple IEDs, each connected to its own GPS receiver, is required [7]. Natural interference (e.g. Solar Flares) is also considered to have negative effects on the GPS signal reception [8] and a GPS satellite anomaly [9] interrupted the 1-PPS output on almost all the GPS receivers used in several substations in Japan. For obvious reasons, there is no legitimate commercially available GPS spoofer, although researchers have built their own and successfully mislead critical devices that rely on a GPS signal, such as Phasor Measurement Unit (PMU) [10]. As a consequence of these fears and greater awareness of threats to critical infrastructure, a time synchronization system that uses fewer, but more reliable, GPS receivers is preferred by utilities.

Synchronization based on the IEEE 1588-2008 standard [11] can be implemented over Ethernet and realizes sub micro-second accuracy when 1588 compliant hardware is used. A substation now requires two or three 1588 master clocks in conjunction with data network, instead of using a local GPS receiver with each IED [12]. The 1588 traffic shares the Ethernet with IEC 61850 Sampled Value (SV) [13] and Generic Object Oriented Substation Event (GOOSE) [14] applications, which allows the use of a unified network consisting of 1588 clocks, 61850 Merging Units (MUs) and IEDs [12] [15]. In the future, substation timing or synchronizing systems will fall into two main categories [12] as shown in Fig. 1(a) and 1(b). The former consists of a network of point-to-point connections that carry 1-PPS / IRIG-B information to a small number of 61850 devices. Whilst the latter involves a large number of 61850 devices and a network of 1588 compliant Ethernet switches shared by IEDs, MUs and 1588 slaves that convert 1588 to 1-PPS / IRIG-B.

The performance of the IEEE 1588 technology, in terms of 1588 device characteristics, were assessed in [16] and the impact of different data networks conditions analyzed in [3], [17]-[18]. When 1588 timing is needed within a harsh substation environment, it must be both reliable and sufficiently robust so that critical automation devices relying on synchronization will not mal-operate. The IEEE 1588 Power 
Profile is defined by the IEEE C37.238-2011 standard [19] for the power system applications and specifies a number of fixed features so that interoperability and predictable performance can be obtained. Ingram et al. conducted comprehensive tests on a network using the 1588 Power Profile in conjunction with $61850 \mathrm{SV}$ and GOOSE applications; the results were used to assess the timing performance in terms of the 1-PPS delay, message compensation accuracy and system reliability [2] [20]-[21].

(a)

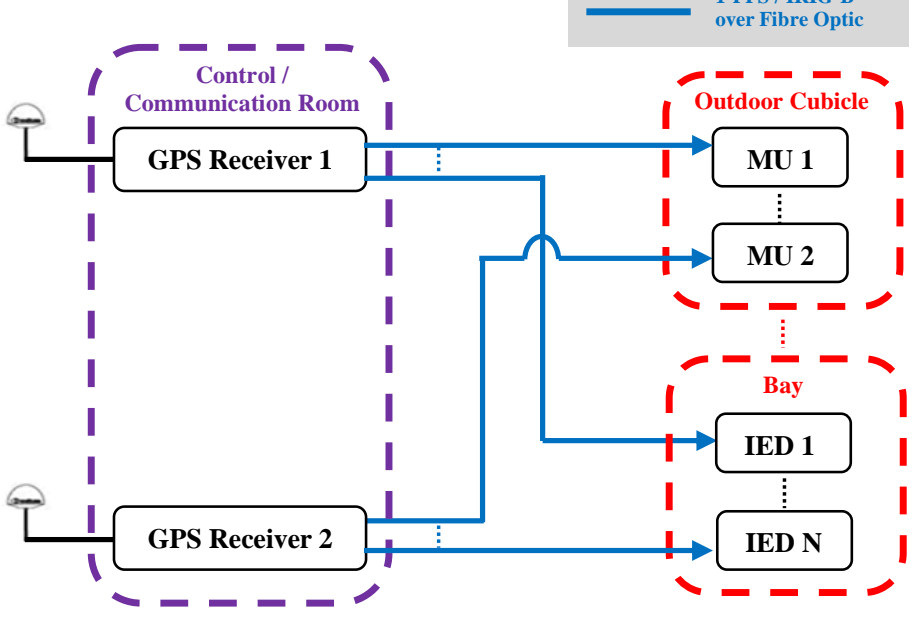

(b)

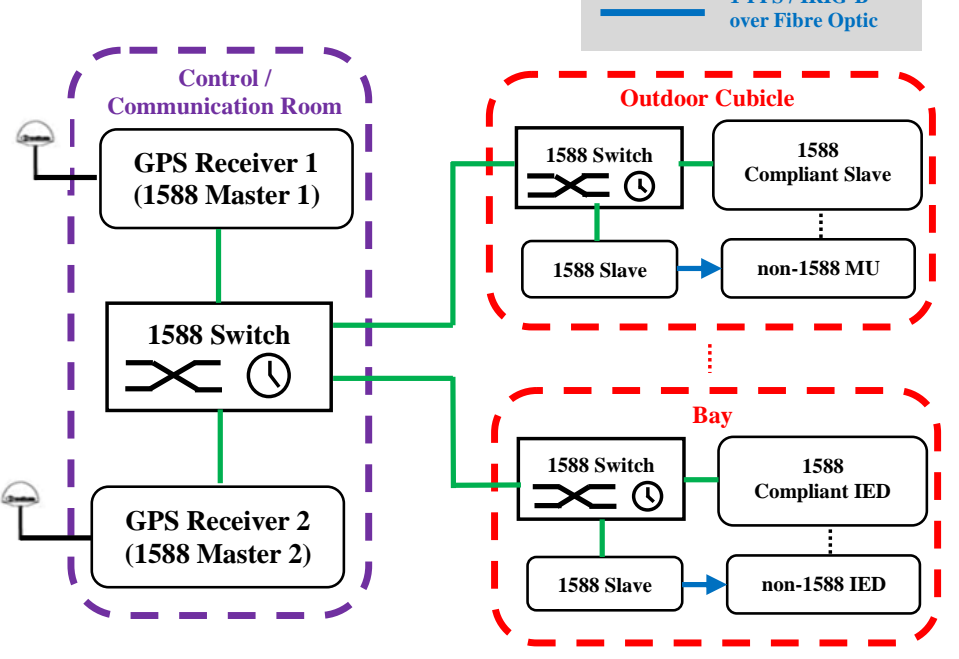

Fig. 1. Timing System based on (a) local GPS receivers and (b) a mixture of GPS receivers and IEEE 1588 devices.

Synchronization test results on the GPS receivers used in substations are rarely published, despite the usual "quoted accuracy" of \pm 100 ns. This paper, will evaluate the long term accuracy of various GPS receivers and their transient behavior during satellite signal loss and restoration, and extend the work described in [2] and [20], by considering the effect of:- network topologies, excessive 1588 traffic, 1588 packet loss, communication link loss and complete GPS signal loss. The test setups and methods are described in Section II. Whilst Section III presents and discusses the test results and Section IV describes the conclusions.

\section{EXPERIMENT SETUP AND TEST METHODS}

To assess the timing accuracy, the 1-PPS output from different timing devices are directly compared, as discussed previously in [2]-[3], [20]. However, instead of using an oscilloscope to measure the time difference, a Linux server with 22 peripheral 1-PPS input channels is employed as shown in Fig. 2. The server can simultaneously compare the time of each 1-PPS signal with the reference 1-PPS; the pulse delay values are plotted in real time and then recorded in a text file for later analysis. A 24 hour measurement file that contains 86,400 records for each input channel, consumes $1.7 \mathrm{MB}$ of disk space; hence, the long term accuracy of a timing device can be easily monitored and analyzed.

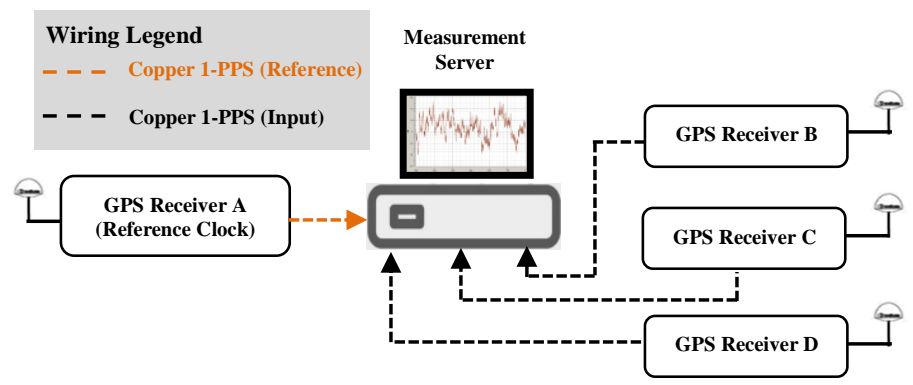

Fig. 2. Experiment Setup for Synchronization Assessment of GPS Receivers.

\section{A. Accuracy Validation of Measurement Server}

Delay measurements are made by the server; which requires the validation of the measurement accuracy. Hence, the 1-PPS output of a GPS receiver is split and connected to the reference port and the input port using copper cables of identical length. The validation procedure for each channel is run for 1000 seconds, i.e. 1000 pulse delay values are recorded. Ideally, the pulse delay should be 0 ns since the same 1-PPS is compared and the propagation delay from the GPS receiver to the server is identical. However, measurement error is inevitable and as illustrated in Table I the error caused by the server is $< \pm 7 \mathrm{~ns}$, which is negligible as compared to the pulse delay accuracy requirements of a substation.

TABLE I

ERROR OF MEASUREMENT SERVER

\begin{tabular}{cccc}
\hline \hline Average & Standard Deviation & Maximum & Minimum \\
\hline $1.392 \mathrm{~ns}$ & $2.147 \mathrm{~ns}$ & $7 \mathrm{~ns}$ & $-6 \mathrm{~ns}$ \\
\hline \hline
\end{tabular}

\section{B. Requirement for Synchronization Accuracy}

When designing a timing system, applications requiring a time reference have to be identified and the associated accuracy requirements determined. The de facto implementation of $\mathrm{SV}$ is specified by the 61850-9-2 Light Edition [24] and the required accuracy of a synchronization source (i.e. devices providing 1 -PPS) is $< \pm 1 \mu \mathrm{s}$. Authors in [22] [25] also suggest $\pm 1 \mu \mathrm{s}$ timing accuracy for Phasor Measurement Units (PMUs). Consequently, $\pm 1 \mu \mathrm{s}$ is the performance baseline used in this paper.

\section{Long Term Synchronization Accuracy of GPS Receivers}

The long term timing accuracy of three different GPS receivers " $\mathrm{B}, \mathrm{C}$ and $\mathrm{D}$ " is assessed by measuring the 1-PPS delay over a 24 hour period for each of the receivers, using the experimental setup in Fig. 2. The mask angle in the 
configuration of Receiver $\mathrm{B}$ can be raised to ensure low-elevation satellites seen by the antenna can be excluded [24]; this reduces the timing error due to signal multipath propagation. To investigate this effect, the 24 hour pulse delay measurement of GPS Receiver B is repeated at different mask angle configurations $\left(5^{\circ}, 15^{\circ}\right.$ and $\left.25^{\circ}\right)$.

\section{Transient Behavior of GPS Receivers}

When using GPS receivers, it is possible for the GPS signal to disappear and recover later. Previous research has indicated the 1-PPS of certain GPS receivers were mistimed during GPS re-synchronization [2] [4]. This emphasizes the importance of assessing transient behavior of receivers during GPS loss and restoration. This involves disconnecting and re-connecting the GPS antenna and monitoring the effect on the pulse delay using the setup in Fig. 2.

\section{E. Long Term Synchronization Accuracy of 1588Slaves}

The long term timing accuracy of two 1588 slaves were assessed by comparing their 1-PPS to the reference 1-PPS over a 24 hour monitoring period, as indicated in Fig. 3.

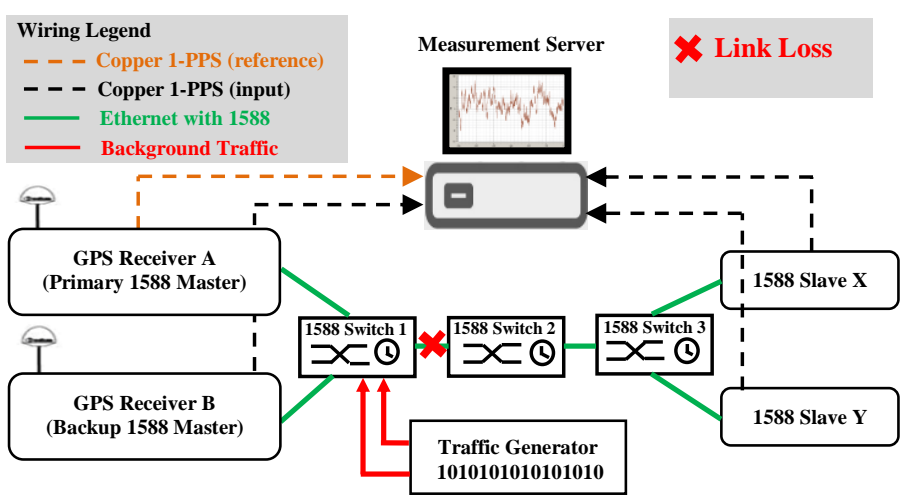

Fig. 3. Synchronization Assessment of 1588 Slaves using Star Topology.

To provide a benchmark for the 1588 synchronization assessment, Receiver A was directly connected to each of the 1588 Slaves. In real applications, various communication architectures are deployed between the GPS receivers and the 1588 slaves. The four topologies commonly used in substation automation systems are assessed in this paper, i.e. Star connection, Rapid Spanning Tree Protocol (RSTP) [27] ring, Parallel Redundancy Protocol (PRP) [28] network and High-available Seamless Ring (HSR) [28]; see Fig 3 and Fig. 4.

Each 1588 device uses a two-step operation mode where the 1588 Sync and Follow_Up messages appear together. Furthermore, all the Ethernet switches are from the same manufacturer and each is configured as a Transparent Clock (TC) with Peer-to-Peer mechanism as specified by the 1588 Power Profile. Receiver B, with a $25^{\circ}$ mask angle, is configured as the backup 1588 master clock if Receiver A degrades or fails. The 1588 Power Profile also specifies the worst-case timing error, this should not exceed $\pm 1 \mu$ s when the network load occupies up to $80 \%$ of the total bandwidth [21]. To investigate the impact of background traffic, the delay measurement is conducted over 24 hours without and with traffic injection. Since the 1588 synchronization shares the data network with 61850 SV and GOOSE applications, a significant portion of the network load will be multicast traffic. The 61850 standards propose the use of IEEE 802.1Q tagging for traffic prioritization, with a default value of 4 [14] [15]. Hence, a traffic generator is utilized to inject $80 \%$ multicast non- 1588 traffic with priority 4 at the leftmost Ethernet switch in all the topologies. The bandwidth of each Ethernet switch is $100 \mathrm{Mb} / \mathrm{s}$ and the 1588 traffic uses priority 4 as defined in the 1588 Power Profile.

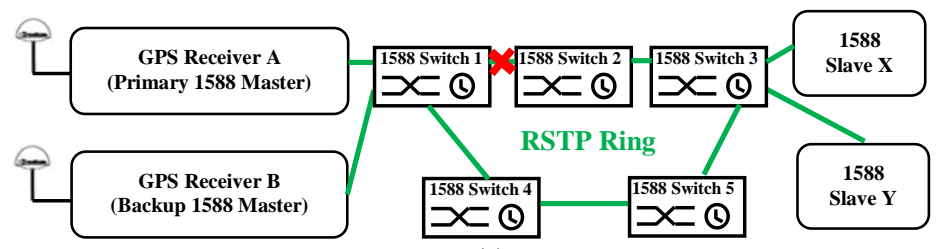

(a)

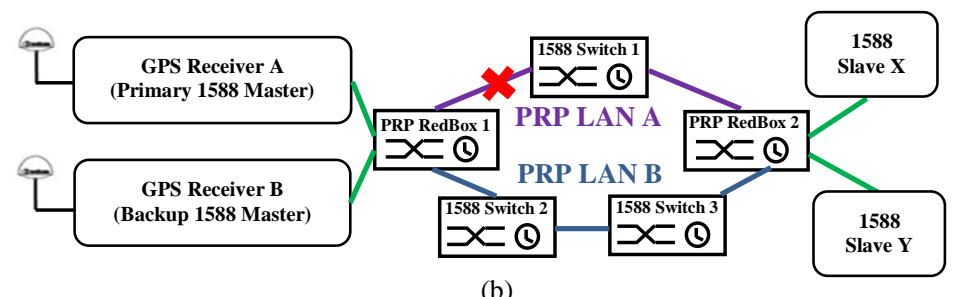

(b)

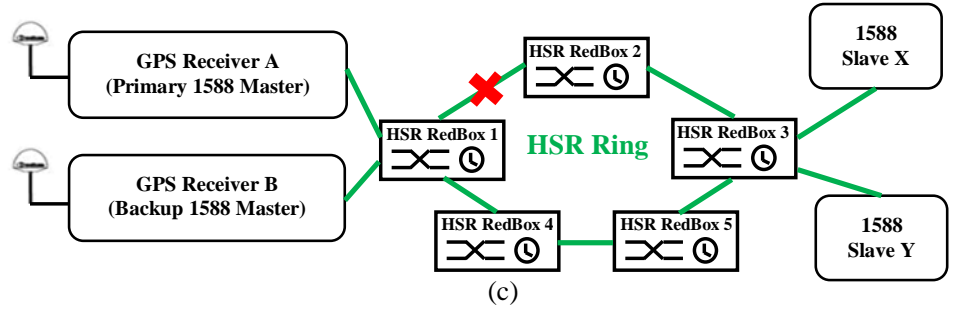

Fig. 4. Network Topologies used between GPS Receivers and 1588 Slaves.

\section{F. 1588 Synchronization under Excessive Network Load}

If a 1588 timing network is not carefully designed and engineered, excessive traffic could appear which may adversely affect synchronization accuracy and result in mal-operation of critical protection and control applications, such as feeder differential protection or PMU based wide area control. Consequently, it is essential to overload the network to verify the effect on the timing performance. Two types of network load are injected into the HSR topology during the tests, namely non-1588 traffic and 1588 traffic.

For non-1588 traffic, the traffic generator injects into the leftmost Ethernet switch:- 100\%, 160\% and 200\% multicast traffic with priority 4 for 1000 seconds. With regard to 1588 traffic, the setup is shown in Fig. 5, where Slaves $\mathrm{X}$ and $\mathrm{Y}$ are synchronized by Receiver B, whilst Receiver A with two 1588 ports operates as the traffic generator and provides up to 128 Sync and Follow_Up messages per second. The Best Master Clock Algorithm (BMCA) defined in 1588 can select the best clock in the network as the primary master, whilst the other potential master(s) enters the "Passive" state and does not send 1588 Sync and Follow_Up messages. To force Receiver A to inject 1588 traffic when it is not the primary master, it is configured to accept only 1588 Announce packets with 802.1Q VLAN tag. In the HSR RedBox 1, the VLAN tags of all the 1588 packets transmitted to Receiver A are set to be stripped. Consequently, Receiver A does not know there is a better clock Receiver B and regards itself as the primary master, transmitting Sync and Follow_Up packets. The 1-PPS of Slave 
$\mathrm{X}$ and $\mathrm{Y}$ is compared to Receiver A during non-1588 traffic injection and to Receiver B when injecting 1588 traffic.

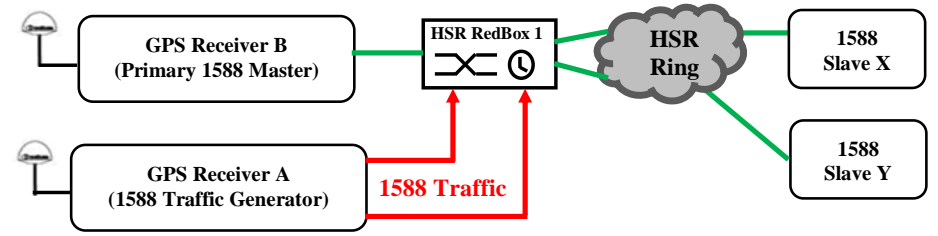

Fig. 5. Injection of 1588 Traffic in HSR Ring Topology.

\section{G. Impact of 1588 Message Loss on 1588 Slaves}

1588 slaves rely on the receipt of Sync and Follow_Up messages for time synchronization. However, Ethernet frames can occasionally be discarded due to network contingencies. Hence, it is important to assess how 1588 slaves will respond to the loss of Sync and Follow_Up messages, which is achieved using the traffic impairment emulator, connected between Receiver A and the slaves; see Fig. 6. The 1-PPS of both 1588 slaves is compared with the 1-PPS of Receiver A.

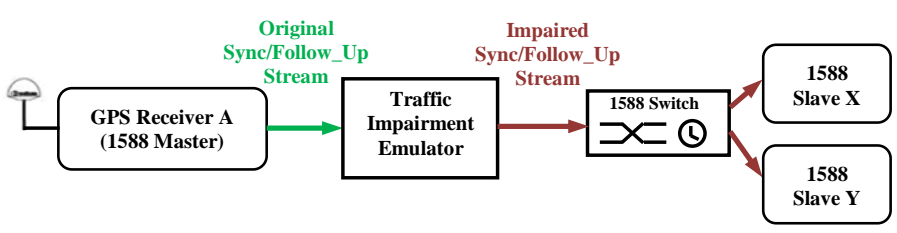

Fig. 6. Emulation of 1588 Message Loss.

\section{H. 1588 Synchronization during Communication Link Loss}

Communication link loss often occurs in substations, which requires $\mathrm{P} \& \mathrm{C}$ systems to use communication links with an appropriate level of redundancy. Following a link loss, RSTP ring topology, which is widely applied in substations, can restore communications within 50 to $2000 \mathrm{~ms}$ [3] [27]. In comparison, a PRP LAN or a HSR ring delivers seamless redundancy $(0 \mathrm{~s}$ recovery time $)$, but at present, these are not widely used in substations. Prior to the deployment of 1588 timing in substations, it is crucial to assess the impact of a link loss on synchronization, and to ensure the network architecture(s) can build a robust 1588 system. Link loss, as denoted by the red cross, is introduced to all the topologies described in Fig. 3 and Fig. 4, and 80\% background traffic is injected during the link loss to produce more realistic results.

\section{Impact of Complete GPS Signal Loss on 1588 Slaves}

For the system shown in Fig. 3, degradation of the time quality of the Primary 1588 Master, will force the Slaves to track the Backup Master which now has a higher time quality than the Primary Master. Assuming, there is minimal offset between the internal oscillators of both masters, the timing accuracy of the Slaves will not be significantly affected [21]. However, in an extreme situation where GPS is lost for all 1588 masters, the impact on the 1588 slaves needs to be investigated. A star topology with $80 \%$ non-1588 background traffic, as shown in Fig. 3 was used and the GPS antennas were first disconnected from Receiver A and then Receiver B. Note: Receiver $\mathrm{C}$ is used as the reference for this test.

\section{TEST RESULtS AND DISCUSSIONS}

\section{A. Long Term Synchronization Accuracy of GPS Receivers}

The 24 hour 1-PPS delay measurement for GPS Receivers B, C and D are plotted in Fig. 7 and Fig. 8; with detailed statistics of timing accuracy summarized in Table II. The average delay value is denoted as $\bar{\alpha}$ whilst the standard deviation is denoted as $\sigma$ in Table II.

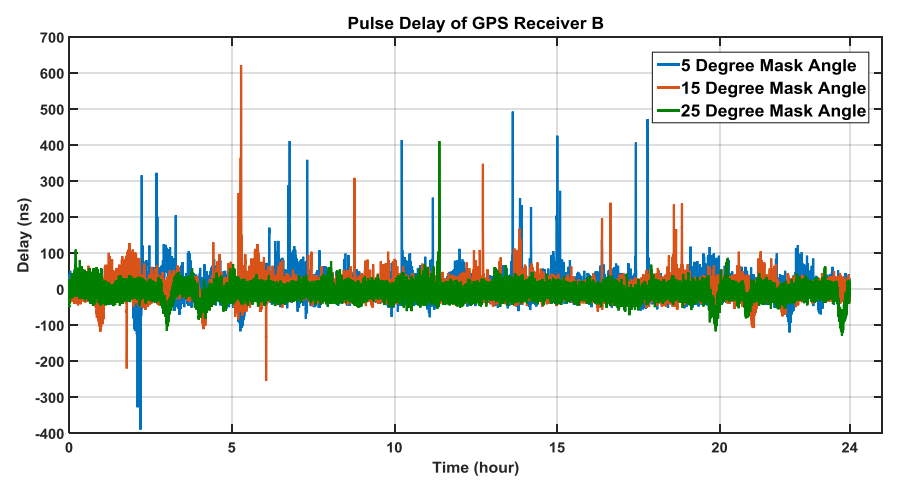

Fig. 7. Long Term Synchronization Accuracy of GPS Receiver B.

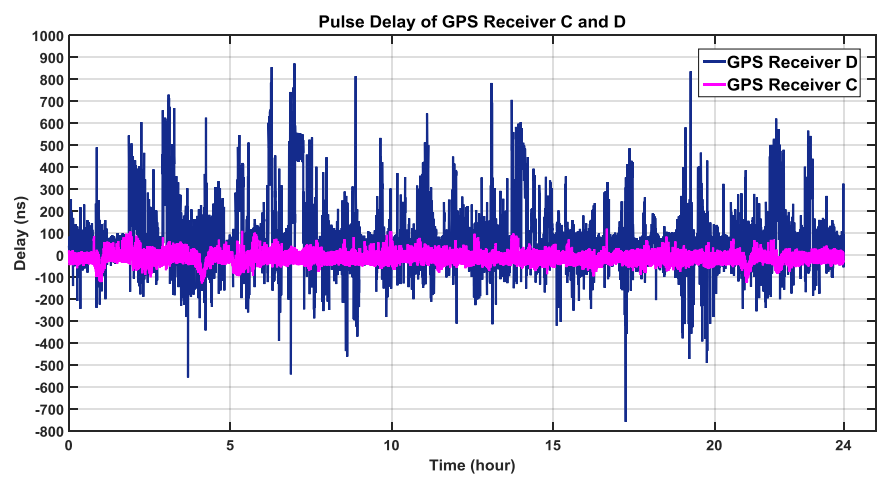

Fig. 8. Long Term Synchronization Accuracy of GPS Receiver C and D.

TABLE II

LONG TERM SYNCHRONIZATION ACCURACY OF GPS RECEIVERS

\begin{tabular}{|c|c|c|c|}
\hline GPS Receiver & $\overline{\bar{\alpha}}$ & $\bar{\sigma}$ & Range \\
\hline B with $5^{\circ}$ Mask Angle & $3.448 \mathrm{~ns}$ & $30.911 \mathrm{~ns}$ & $-390 \mathrm{~ns}$ to $493 \mathrm{~ns}$ \\
\hline B with $15^{\circ}$ Mask Angle & $1.287 \mathrm{~ns}$ & $26.166 \mathrm{~ns}$ & $-255 \mathrm{~ns}$ to $622 \mathrm{~ns}$ \\
\hline B with $25^{\circ}$ Mask Angle & $-8.036 \mathrm{~ns}$ & $19.62 \mathrm{~ns}$ & $-129 \mathrm{~ns}$ to $411 \mathrm{~ns}$ \\
\hline $\mathrm{C}$ & $-10.532 \mathrm{~ns}$ & $23.449 \mathrm{~ns}$ & $-128 \mathrm{~ns}$ to $121 \mathrm{~ns}$ \\
\hline $\bar{D}$ & $80.906 \mathrm{~ns}$ & $126.756 \mathrm{~ns}$ & $-758 \mathrm{~ns}$ to $873 \mathrm{~ns}$ \\
\hline
\end{tabular}

Test results indicate Receiver $\mathrm{C}$ has the most stable 1-PPS output and can deliver timing accuracy better than $\pm 150 \mathrm{~ns}$. Fig. 7 suggests increased mask angle reduces the occurrence of delay spikes and helps maintain the accuracy of Receiver B within $\pm 420 \mathrm{~ns}$. Without a configurable mask angle, Receiver D can only deliver timing accuracy in the range of $\pm 880 \mathrm{~ns}$, with a standard deviation of $127 \mathrm{~ns}$. In comparison with Receiver C, Receivers B and D experience many delay spikes, which are probably caused by GPS multipath propagation resulting from a high wall to the South of the GPS antennas, as well as the close proximity of the antennas [28], as shown in Fig. 9. Further work is required to verify this observation; this involves moving the GPS antennas to a location where they can be installed far apart and with no obstruction to the sky.

In conclusion, Receiver $\mathrm{C}$ is the best for $\mathrm{SV}$ and phasor measurements because of its high accuracy and immunity to 
multipath problems. Receiver B can also satisfy the $\pm 1 \mu \mathrm{s}$ requirement with an appropriate mask angle configuration, but it may not be advisable to use Receiver D for accurate timing.

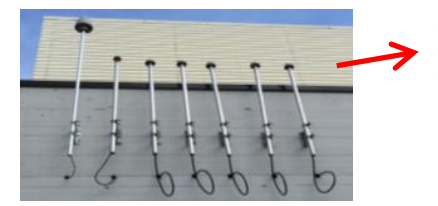

High Wall to the South of GPS Antennas

Fig. 9. Installation and Position of GPS Antennas.

\section{B. Transient Behavior of GPS Receivers}

Test results for the transient behavior of GPS receivers are shown in Fig. 10 and 11 and all the incidents occur at $\mathrm{t}=100 \mathrm{~s}$. (i) Loss of GPS Signal

Fig. 10 shows Receiver C stops outputting 1-PPS once it detects loss of the GPS signal, as illustrated by the blue line at the zero axis. However, Receiver B and D continue to generate 1-PPS after GPS loss and exhibit drifting rates with diverse signs and magnitudes. Receiver B usually drifts positively at an approximate rate $2.22 \mathrm{~ns} / \mathrm{s}$ as indicated by the orange curve. Although rarer, Receiver B can also drift in the negative direction at $1.61 \mathrm{~ns} / \mathrm{s}$, according to the amber plot. Hence, with a worst case initial delay value of $622 \mathrm{~ns}$, Receiver B reaches the $\pm 1 \mu$ s limit after $170 \mathrm{~s}$. Similarly, Receiver D always drifts negatively, as shown by the purple line, and its slope varies between $-1.25 \mathrm{~ns} / \mathrm{s}$ and $0.57 \mathrm{~ns} / \mathrm{s}$. Receiver D breaks the $\pm 1 \mu \mathrm{s}$ limit after $193 \mathrm{~s}$, assuming a worst case initial delay value of -758 ns. When considering these results, it is important to recognize that most MUs and IEDs can detect the loss of the synchronization input and in turn inhibit operation [29] [30]. Therefore, Receiver C, which stops outputting 1-PPS when GPS is lost, is better than Receiver B and D, since relay mal-operation can be avoided.

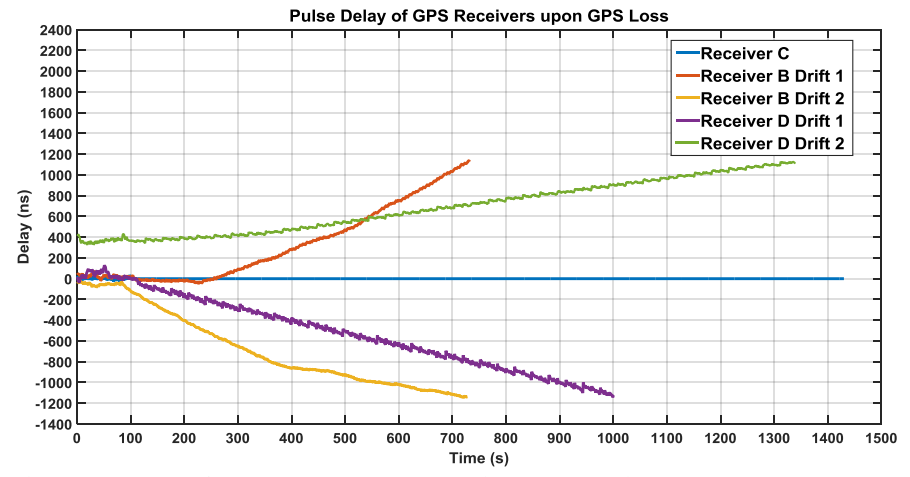

Fig. 10. Impact of GPS Loss on GPS Receiver B, C and D.

\section{(ii) Restoration of GPS Signal}

Fig. 11 shows the effect of GPS restoration on receivers B, $\mathrm{C}$ and D. When B regains GPS, the 1-PPS delay suddenly increases from $200 \mathrm{~ns}$ to $-3,800 \mathrm{~ns}$, which is not acceptable for most types of IEDs. In comparison, the delay change is negligible when C re-synchronizes to GPS, and small ( $<400 \mathrm{~ns}$ ) when D re-synchronizes. Consequently, Receivers C and D can guarantee correct operation of MUs, IEDs and PMUs.

The transient behavior of Receiver $B$ indicates it may severely break the $\pm 1 \mu$ s requirement during GPS restoration and is only able to maintain micro-second accuracy for about $\approx 500 \mathrm{~s}$ after the loss of GPS. Similarly, D can only maintain \pm 1 $\mu$ s accuracy for $\approx 700 \mathrm{~s}$ following loss of GPS, but it does work correctly during GPS resynchronization. Receiver C provides accurate timing during GPS restoration and stops outputting 1-PPS when it detects loss of GPS.

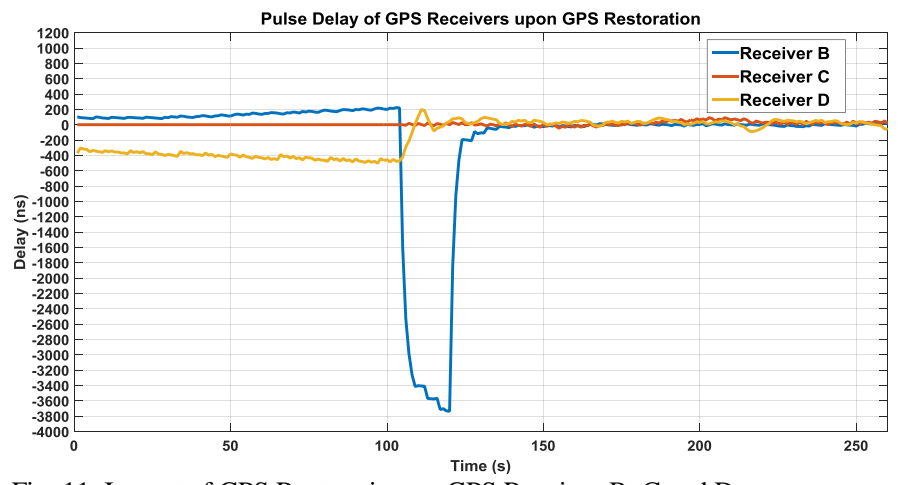

Fig. 11. Impact of GPS Restoration on GPS Receiver B, C and D.

\section{Long Term Synchronization Accuracy of 1588 Slaves}

Timing accuracy varies from slave to slave and may be affected by different network topology and load conditions. Statistics of 1-PPS delay of Slave X compared to Receiver A is summarized in Table III whilst details of Slave Y are covered in Table IV.

TABLE III

LONG TERM SyNCHRONIZATION ACCURACY OF SLAVE X

\begin{tabular}{c|c|c|c}
\hline \hline \multicolumn{1}{c}{ Topology and Traffic } & $\overline{\boldsymbol{x}}$ & $\boldsymbol{\sigma}$ & \multicolumn{1}{c}{ Range } \\
\hline Direct & $-8.688 \mathrm{~ns}$ & $8.064 \mathrm{~ns}$ & $-32 \mathrm{~ns}$ to $22 \mathrm{~ns}$ \\
\hline Star without Traffic & $-11.482 \mathrm{~ns}$ & $29.634 \mathrm{~ns}$ & $-107 \mathrm{~ns}$ to $73 \mathrm{~ns}$ \\
\hline Star with 80\% Traffic & $-9.401 \mathrm{~ns}$ & $38.105 \mathrm{~ns}$ & $-125 \mathrm{~ns}$ to $113 \mathrm{~ns}$ \\
\hline RSTP without Traffic & $7.069 \mathrm{~ns}$ & $31.987 \mathrm{~ns}$ & $-87 \mathrm{~ns}$ to $113 \mathrm{~ns}$ \\
\hline RSTP with 80\% Traffic & $5.46 \mathrm{~ns}$ & $29.611 \mathrm{~ns}$ & $-83 \mathrm{~ns}$ to $88 \mathrm{~ns}$ \\
\hline PRP without Traffic & $11.998 \mathrm{~ns}$ & $23.592 \mathrm{~ns}$ & $-63 \mathrm{~ns}$ to $90 \mathrm{~ns}$ \\
\hline PRP with 80\% Traffic & $11.32 \mathrm{~ns}$ & $27.869 \mathrm{~ns}$ & $-67 \mathrm{~ns}$ to $98 \mathrm{~ns}$ \\
\hline HSR without Traffic & $29.412 \mathrm{~ns}$ & $18.946 \mathrm{~ns}$ & $-38 \mathrm{~ns}$ to $95 \mathrm{~ns}$ \\
\hline HSR with 80\% Traffic & $30.024 \mathrm{~ns}$ & $18.389 \mathrm{~ns}$ & $-39 \mathrm{~ns}$ to $82 \mathrm{~ns}$ \\
\hline \hline
\end{tabular}

TABLE IV

LONG TERM SYNCHRONIZATION ACCURACY OF SLAVE Y

\begin{tabular}{c|c|c|c}
\hline \hline \multicolumn{1}{c}{ Topology and Traffic } & $\bar{y}$ & \multicolumn{1}{c}{$\boldsymbol{\sigma}$} & Range \\
\hline Direct & $-13.047 \mathrm{~ns}$ & $9.547 \mathrm{~ns}$ & $-43 \mathrm{~ns}$ to $16 \mathrm{~ns}$ \\
\hline Star without Traffic & $-14.959 \mathrm{~ns}$ & $18.041 \mathrm{~ns}$ & $-66 \mathrm{~ns}$ to $46 \mathrm{~ns}$ \\
\hline Star with 80\% Traffic & $-10.324 \mathrm{~ns}$ & $27.766 \mathrm{~ns}$ & $-93 \mathrm{~ns}$ to $74 \mathrm{~ns}$ \\
\hline RSTP without Traffic & $4.761 \mathrm{~ns}$ & $21.071 \mathrm{~ns}$ & $-60 \mathrm{~ns}$ to $68 \mathrm{~ns}$ \\
\hline RSTP with 80\% Traffic & $0.501 \mathrm{~ns}$ & $18.572 \mathrm{~ns}$ & $-56 \mathrm{~ns}$ to $71 \mathrm{~ns}$ \\
\hline PRP without Traffic & $9.769 \mathrm{~ns}$ & $15.55 \mathrm{~ns}$ & $-46 \mathrm{~ns}$ to $63 \mathrm{~ns}$ \\
\hline PRP with 80\% Traffic & $11.191 \mathrm{~ns}$ & $18.462 \mathrm{~ns}$ & $-53 \mathrm{~ns}$ to $74 \mathrm{~ns}$ \\
\hline HSR without Traffic & $62.149 \mathrm{~ns}$ & $15.204 \mathrm{~ns}$ & $0 \mathrm{~ns}$ to $122 \mathrm{~ns}$ \\
\hline HSR with 80\% Traffic & $63.166 \mathrm{~ns}$ & $14.829 \mathrm{~ns}$ & $14 \mathrm{~ns}$ to $117 \mathrm{~ns}$ \\
\hline \hline
\end{tabular}

In general, Slave $X$ and Slave $Y$ can both deliver accuracy much better than $\pm 1 \mu$ s (i.e. Slave $X= \pm 125$ ns; Slave $Y= \pm 122$ ns) even when $80 \%$ of the bandwidth is occupied by other equal priority traffic. The introduction of 1588 Ethernet switches will increase the standard deviation of the 1-PPS delay; they introduce errors when calculating the path delay and residence time, but the increase is negligible ( $<35 \mathrm{~ns})$. The Star topology has the least impact on the timing accuracy, whilst HSR topology has the greatest. This is because the HSR RedBox connected to the 1588 master acts as a Boundary Clock (BC), even if it is configured as a TC. Increased errors are also present during the process where a $\mathrm{BC}$ terminates the 1588 
packets and regenerates the 1588 flow; this involves synchronizing to the upstream Master and disciplining the downstream Slaves. Multicast traffic does not greatly affect the timing performance but the primary 1588 master (Receiver A) will stop working when $>25 \mathrm{Mb} / \mathrm{s}$ traffic appears on its port. Hence, multicast traffic not needed by the master should be filtered out using the MAC address and/or the VLAN setting in the Ethernet switch.

\section{1588 Synchronization under Excess Network Load}

\section{(i) Non-1588 Traffic}

Timing accuracy of Slave $\mathrm{X}$ and $\mathrm{Y}$ during excessive level of non-1588 multicast traffic is listed in Table V. Although the network is extremely congested, both Slave X and Y obtain accuracy $< \pm 120 \mathrm{~ns}$. Theoretically, when a large amount of traffic with the same priority competes for transmission, 1588 packets will experience additional delay. If the delay is not properly measured by 1588 switches, the accuracy could be severely affected. Furthermore, excessive network traffic can fill the switch buffer and 1588 packets may be discarded when the buffer is full, leading to unexpected mistiming. In fact, the 1588 switches used during the experiment are able to preserve the transmission of 1588 packets even when non-1588 traffic with higher priority is injected. Results in Table $\mathrm{V}$ also indicate Ethernet switches accurately measure the delay experienced by 1588 packets even if the non-1588 load is excessive.

TABLE V

TIMING ACCURACY OF SLAVES UNDER EXCESSIVE NON-1588 TRAFFIC

\begin{tabular}{|c|c|c|c|}
\hline Slave and Traffic & $\overline{\bar{\alpha}}$ & $\bar{\sigma}$ & Range \\
\hline X, $100 \%$ Traffic & $34.74 \mathrm{~ns}$ & $18.75 \mathrm{~ns}$ & $-35 \mathrm{~ns}$ to $85 \mathrm{~ns}$ \\
\hline $\mathrm{X}, 160 \%$ Traffic & $30.985 \mathrm{~ns}$ & $18.36 \mathrm{~ns}$ & $-35 \mathrm{~ns}$ to $79 \mathrm{~ns}$ \\
\hline $\mathrm{X}, 200 \%$ Traffic & $34.895 \mathrm{~ns}$ & $19.124 \mathrm{~ns}$ & $-25 \mathrm{~ns}$ to $91 \mathrm{~ns}$ \\
\hline Y, 100\% Traffic & $64.148 \mathrm{~ns}$ & $16.128 \mathrm{~ns}$ & $17 \mathrm{~ns}$ to $113 \mathrm{~ns}$ \\
\hline Y, 160\% Traffic & $61.435 \mathrm{~ns}$ & $14.945 \mathrm{~ns}$ & $12 \mathrm{~ns}$ to $102 \mathrm{~ns}$ \\
\hline Y, 200\% Traffic & $63.576 \mathrm{~ns}$ & $16.056 \mathrm{~ns}$ & $17 \mathrm{~ns}$ to $117 \mathrm{~ns}$ \\
\hline
\end{tabular}

\section{(ii) 1588 Traffic}

Fig. 12 shows Slave $\mathrm{X}$ and $\mathrm{Y}$ correctly synchronize to Receiver B within $100 \mathrm{~s}$ when Receiver A only injects 96 Sync and Follow_Up messages per second. After 100 s, 128 Sync and Follow_Up packets are injected into the network every second and the slaves start to drift away.

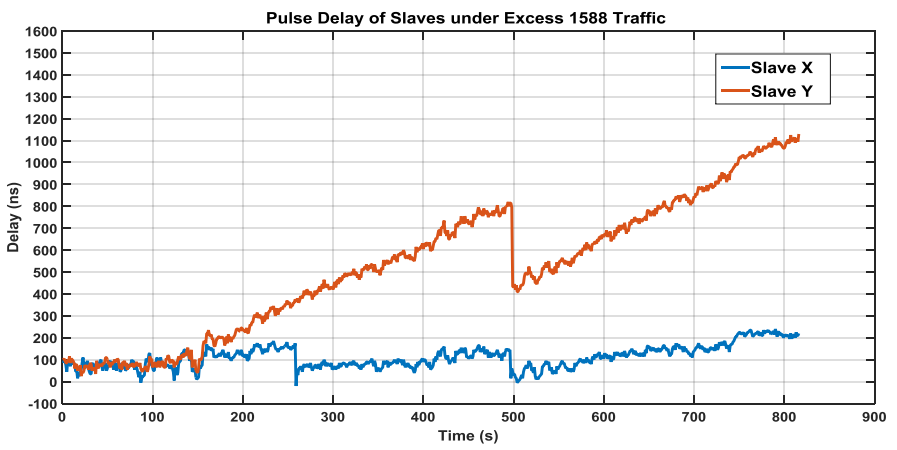

Fig. 12. Impact of Excess 1588 Traffic on 1588 Timing.

The Wireshark capture indicates the 1588 switches cannot process this many 1588 Sync packets and most of the Sync and Follow_Up originated from Receiver B are discarded. Hence, the slaves keep losing synchronization and the clocks drift. However, when some 1588 packets from Receiver B are not dropped, the slaves use these packets to adjust their internal oscillators, leading to the sudden decreases in pulse delay as shown in Fig. 12. Instead of using the VLAN setting to force Receiver A to inject 1588 traffic when it is not the best clock, if the Ethernet switch can forward 1588 packets with different domain numbers, Receiver A can also inject 1588 packets to stress the network. Consequently, the 1588 network should be carefully configured to ensure it is not breached by excessive 1588 traffic resulting from misconfiguration or a cyber-attack.

\section{E. Impact of 1588 Message Loss on 1588 Slaves}

(i) Loss of 1588 Sync Packets

Test results with various Sync loss rates are plotted in Fig. 13 and the Sync loss begins at $t=100$ s. Slave $X$ can tolerate up to $99 \%$ Sync message loss and can synchronize itself to the master very quickly even if it receives only one Sync message. When the Sync messages are completely not available, Slave X drifts at a rate $-0.368 \mathrm{~ns} / \mathrm{s}$. As mentioned in previous section, the worst case initial error of Slave $X$ can be $\pm 125 \mathrm{~ns}$, giving a margin of $-875 \mathrm{~ns}$. Hence, Slave $X$ will reach the $\pm 1 \mu$ s after 2377 s. If there is a Sync packet available during this period (i.e. Sync available rate $=1 / 2377=0.04 \%$ ), Slave $\mathrm{X}$ obtains synchronization again, so theoretically it can withstand a $99.96 \%$ Sync loss rate.

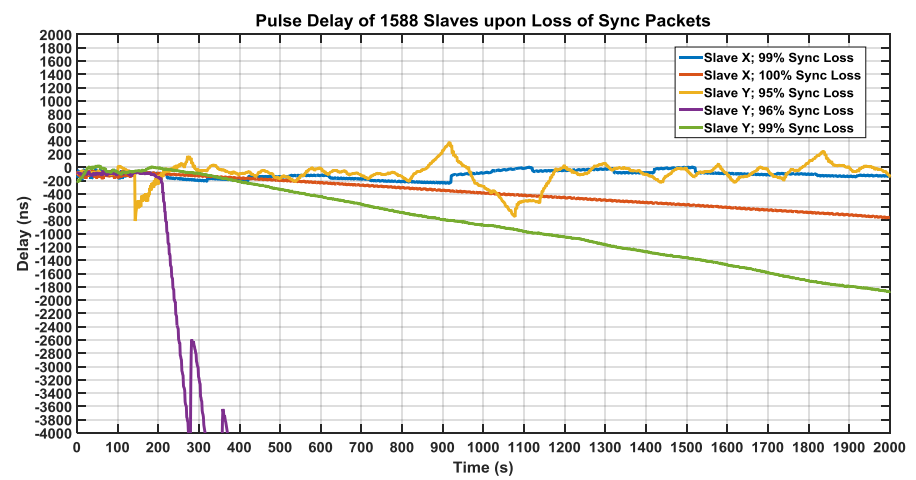

Fig. 13. Impact of 1588 Sync Packet Loss, commencing from $100 \mathrm{~s}$.

Slave Y, can maintain synchronization when 95\% Sync packets are missing but with an error up to $-800 \mathrm{~ns}$. When $96 \%$ Sync packets are discarded, Slave Y loses synchronization within $100 \mathrm{~s}$ and shows an unusual drifting behavior, probably due to its synchronization algorithm. If 99\% Sync packets are dropped, Slave Y drifts at a faster rate $(-0.947 \mathrm{~ns} / \mathrm{s})$ than Slave $\mathrm{X}$; this is because Slave $\mathrm{X}$ includes a more stable Oven Controlled Crystal Oscillator while Slave Y uses a Temperature Controlled Crystal Oscillator. This also suggests the excess 1588 traffic injected in the previous experiment can cause at least $99 \%$ Sync packet loss.

\section{(ii) Loss of 1588 Follow_Up Packets}

Similar to the scenario where Sync messages are dropped, Slave $\mathrm{X}$ is not affected by a Follow_Up packet loss of up to $99 \%$, as shown in Fig. 14. If the Follow_Up packets are not presenting at all, Slave $\mathrm{X}$ will start drifting at a rate similar to that observed when the Sync packets are completely lost.

Slave Y is very sensitive to the loss of Follow_Up packets and the pulse delay will dramatically become larger than $80 \mu \mathrm{s}$ even when a single Follow_Up message is missing. Once the anomaly occurs, it takes about $200 \mathrm{~s}$ for Slave Y to recover, 
which would increase the likelihood of relay mal-operation. Experimental results demonstrate a loss rate lower than $99 \%$ will lead to the anomaly, which might be caused by a bug in the synchronization algorithm. When the loss rate is $\geq 99 \%$, Slave Y exhibits a drifting rate similar to that observed when $\geq 99 \%$ Sync packets are absent. Hence, firmware updates are required for Slave $\mathrm{Y}$ to resolve the synchronization issue related to extreme loss (96\% to 99\%) of Sync and Follow_Up messages.

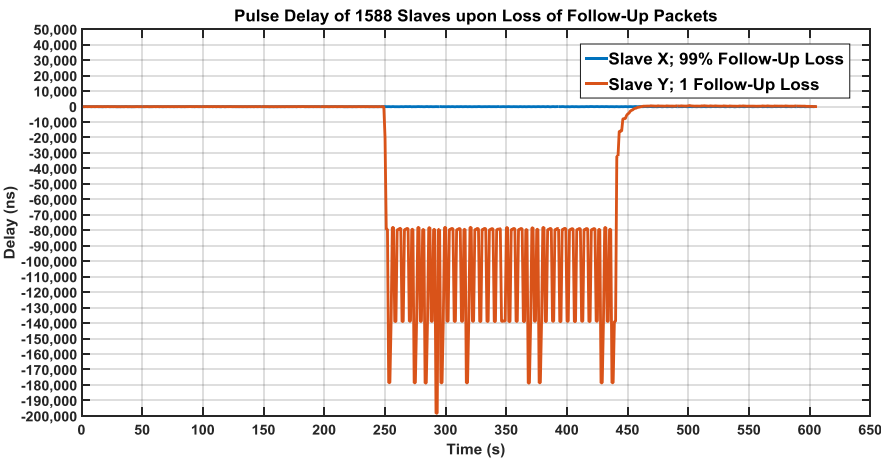

Fig. 14. Impact of 1588 Follow_Up Packet Loss.

\section{F. 1588 Synchronization during Communication Link Loss}

The link loss in Fig. 15 occurs at $\mathrm{t}=100 \mathrm{~s}$, and when Star topology is used Slave X and Y drift away because no 1588 packets from the master are available. When RSTP and HSR topologies are employed, link loss does not affect the timing accuracy, which indicates the 1588 switches and slaves can automatically adjust themselves when swapping to a different communication path. In comparison, link loss in PRP topology introduces considerable fluctuations in pulse delay with a peak value of $50 \mathrm{~ns}$, as indicated by the green curve. However, in general, Slave $\mathrm{X}$ and $\mathrm{Y}$ are able to achieve an accuracy significantly less than $\pm 1 \mu$ s during link loss if communication redundancy is in place.

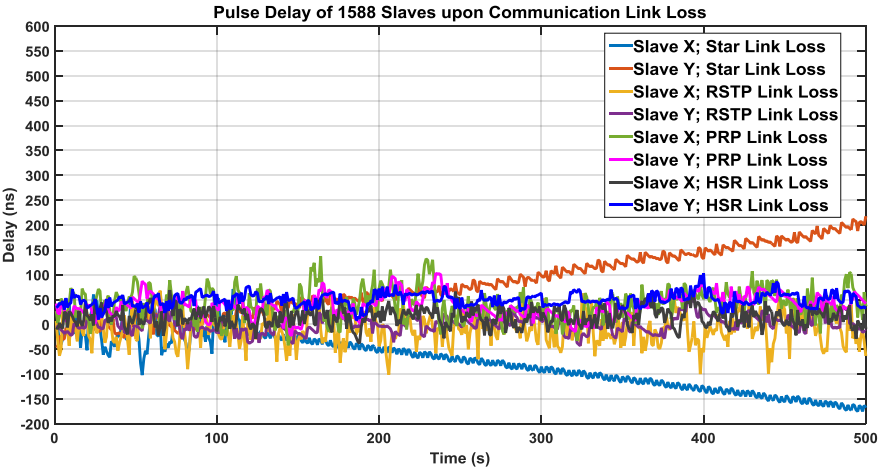

Fig. 15. Impact of Communication Link Loss on 1588 Timing.

\section{G. Impact of Complete GPS Signal Loss on 1588 Slaves}

Fig. 16 illustrates what happens when Slave $\mathrm{X}$ and $\mathrm{Y}$ are initially synchronized to Receiver A and the GPS antenna is disconnected from Receiver A at $\mathrm{t}=200 \mathrm{~s}$. After about $40 \mathrm{~s}$, Receiver B notices that the performance of Receiver A has deteriorated and it takes the master role for the whole network. As a result, Slave $\mathrm{X}$ and $\mathrm{Y}$ start to follow Receiver B with a 200 $\mathrm{ns}$ increase in their pulse delay. At $\mathrm{t}=500 \mathrm{~s}$, the GPS antenna is also disconnected from Receiver B; it continues to act as the master until $\mathrm{t}=540 \mathrm{~s}$ when Receiver A discovers it is a better clock and recovers the master role again. Sequentially, Slave X again synchronizes to Receiver A, but Slave Y drifts away. When Slave Y drifts, its port is in "Slave" state and according to 1588 , it should synchronize to Receiver A once the same pair of Sync and Follow_Up packets is received. The reason the algorithm of Slave Y refuses to synchronize with Receiver A is because the clock accuracy value of Receiver A is "unknown" and it does not satisfy Slave Y's requirement.

Receiver A is more stable than Receiver B when the GPS signal is completely lost and a 1588 slave (e.g. Slave X) follows a stable clock when there is no GPS signal, ensuring the correct operation of the P\&C devices or IEDs. However, if a less stable clock becomes the master after a complete GPS loss, it may be advisable that a 1588 slave, i.e. Slave $\mathrm{Y}$, does not follow the master and issues an alarm so that the associated IEDs can block operation to avoid mal-operation.

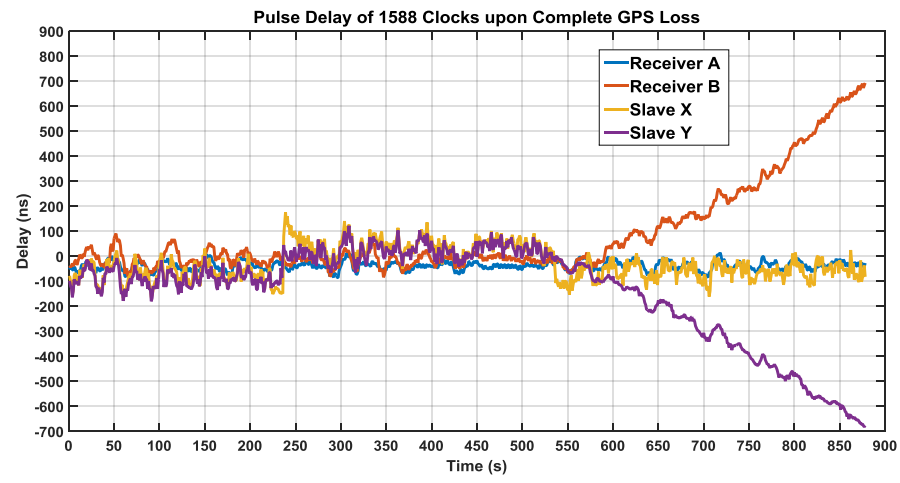

Fig. 16. Pulse Delay between Reference Receiver C and Slaves upon Complete GPS Signal Loss.

\section{CONCLUSION}

Experimental results described in this paper indicate not all GPS receivers can deliver \pm 100 ns accuracy, but an increased mask angle could reduce the timing error. It is advisable that a GPS receiver does not generate any timing output when it loses GPS signal so that the associated P\&C devices or IEDs can block operation to avoid mal-operation. Because of this, system operators may not need to worry about the drifting rate of distributed GPS receivers. One GPS receiver introduced unacceptable synchronization errors, $>3 \mu \mathrm{s}$, upon GPS signal restoration and this has to be resolved to guarantee correct operation of secondary devices.

A data network consisting of 1588 clocks and 1588 Ethernet switches was used to achieve synchronization accuracy better than \pm 150 ns even when the network is heavily loaded. But this requires the use of 1588 Ethernet switches to accurately measure the delay experienced by the 1588 packets and ensure the 1588 messages are not dropped when they need to compete for transmission. This supports the use of a unified IEC 61850 and IEEE 1588 network for future substation automation systems. However, such a network must be carefully designed and configured because considerable non-1588 traffic could "shut down" certain 1588 clocks, whilst excessive 1588 traffic can outreach the processing ability of a 1588 switch, causing loss of synchronization. Communication redundancy techniques can ensure 1588 timing is not affected during communication link loss. However, one 1588 slave could not properly handle the loss of Follow_Up message and introduced timing errors $>80 \mu$ s for about $200 \mathrm{~s}$; this must be fixed by a 
firmware upgrade. 1588 slaves from different vendors use diverse algorithms to deal with the situation where the master does not lock to the GPS - some still synchronize to the master whilst others will not. Therefore, the stability of the 1588 master(s) and the behavior of 1588 slaves during complete GPS loss must be investigated and understood to avoid mal-operation caused by timing drift.

Time synchronization with stringent $\pm 1 \mu$ s accuracy requirement is important for digital substation applications and solutions based on direct use of local GPS receivers have been widely adopted. However, sophisticated tests must be carried out to assess the long term accuracy and transient behavior of GPS receivers during incidents, before they can be considered adequately reliable for transmission substations. The described solution, based on GPS receivers and 1588 devices, is promising as it can be easily integrated into the IEC 61850 process and station buses, and delivers accurate and reliable time synchronization.

\section{REFERENCES}

[1] E. Southern, "GPS Synchronized Current Differential Protection," Ph.D. dissertation, School of EEE, UMIST, 1998.

[2] D. M. E. Ingram, P. Schaub and D. A. Campbell, "Use of Precision Time Protocol to Synchronize Sampled-Value Process Bus," IEEE Trans. Instrum. Meas., vol. 61, no. 5, pp. 1173 - 1180, May 2012.

[3] C. M. De Dominicis, P. Ferrari, A. Flammini, S. Rinaldi and M. Quarantelli, "On the Use of IEEE 1588 in Existing IEC 61850-Based SASs: Current Behavior and Future Challenges," IEEE Trans. Instrum. Meas., vol. 60, no. 9, pp. 3070-3081, Sep. 2011.

[4] W. An, T. Nick, D. Barron, M. Bingham and A. Hackett, "A transmission utility's experience to date with feeder unit protection systems," in Proc. $11^{\text {th }}$ Int. Conf. on Develop. Power Syst. Protection (DPSP), Birmingham, UK, 23-26 Apr. 2012, pp. 1-6.

[5] C. Xu, H. Xiong, L. He, Z. Li and J. Yang, "The Research of Intelligent Substation Time Synchronization System and the Influence of its Fault to Relay Protection," Energy and Power Eng., vol. 5, no. 4B, pp. 468-473, Jul. 2013.

[6] C. Curry. (2014, April). SENTINEL Project - Report on GNSS Vulnerabilities. Chronos Technology, UK. [Online]. Available: www.chronos.co.uk/files/pdfs/gps/SENTINEL Project Report.pdf

[7] B. Baumgartner, C. Riesch and W. Schenk, "GPS Receiver Vulnerabilities Urban Legends or Sad, Hard Truth?" in Proc. PAC World Amer. Conf. 2014, Raleigh, USA, 22-25 Sep. 2014, pp. 1-20.

[8] A. P. Cerruti, P. M. Kintner Jr, D. E. Gary, A. J. Mannucci, R. F. Meyer, P. Doherty and A. J. Coster, "Effect of December 2006 solar radio bursts on GPS receivers" Space Weather, vol. 6, no. 10, pp.1-10, Oct. 2008.

[9] D. Itagaki, K. Ohashi, I. Shuto and H. Ito, "Field Experience and Assessment of GPS Signal Receiving and Distribution System for Synchronizing Power System Protection, Control and Monitoring," in Proc. 2006 IEEE Power India Conf., New Delhi, India, 10-12 Apr. 2006, pp. 1-8.

[10] D. P. Shepard, T. E. Humphreys and A. A. Fansler, "Evaluation of the vulnerability of phasor measurement units to GPS spoofing attacks," Int. J. of Critical Infrastructure Protection, vol. 5, no. 3-4, pp 146-153, Dec. 2012.

[11] IEEE Standard for a Precision Clock Synchronization Protocol for Networked Measurement and Control Systems, IEEE Std 1588-2008, 24 Jul. 2008

[12] Communication networks and systems for power utility automation Part 90-4: Network engineering guidelines, IEC 61850-90-4:2013, Aug. 2013.

[13] Communication networks and systems for power utility automation Part 9-2: Specific communication service mapping (SCSM) - Sampled values over ISO/IEC 8802-3, IEC 61850-9-2:2011, Dec. 2011.

[14] Communication networks and systems for power utility automation Part 8-1: Specific communication service mapping (SCSM) - Mappings to MMS (ISO 9506-1 and ISO 9506-2) and to ISO/IEC 8802-3, IEC 61850-8-1:2011, Sep. 2011.

[15] D. M. E. Ingram, P. Schaub, R. R. Taylor and D. A. Campbell, "System-Level Tests of Transformer Differential Protection Using an
IEC 61850 Process Bus," IEEE Trans. Power Del., vol. 29, no. 3, pp. 1382-1389, Jun. 2014.

[16] L. Cosart, "Characterizing grandmaster, transparent and boundary clocks with a precision packet probe and packet metrics," in Proc. 2011 IEEE Int. Symp. Precis. Clock Synchr. Meas. Control Commun. (ISPCS), Munich, Germany, 12-16 Sep. 2011, pp. 56-61.

[17] R. Harada, A. Abdul and P. Wang, "Best Practice of Transporting PTPv2 over RSTP networks," in Proc. 2012 IEEE Int. Symp. Precis. Clock Synchr. Meas. Control Commun. (ISPCS), San Francisco, USA, 24-28 Sep. 2012, pp. 1-6.

[18] R. Zarick, M. Hagen and R. Bartos, "The Impact of Network Latency on the Synchronization of Real-World IEEE 1588-2008 Devices," in Proc. 2010 IEEE Int. Symp. Precis. Clock Synchr. Meas. Control Commun. (ISPCS), Portsmouth, USA, 27 Sep. - 01 Oct. 2010, pp. 135-140.

[19] IEEE Standard Profile for Use of IEEE $1588^{T M}$ Precision Time Protocol in Power System Applications, IEEE Std C37.238-2011, Jul. 2011.

[20] D. M. E. Ingram, P. Schaub, D. A. Campbell and R. R. Taylor, "Performance Analysis of PTP Components for IEC 61850 Process Bus Applications," IEEE Trans. Instrum. Meas., vol. 62, no. 4, pp. 710-719, Apr. 2013.

[21] D. M. E. Ingram, P. Schaub, D. A. Campbell and R. R. Taylor, "Quantitative Assessment of Fault Tolerant Precision Timing for Electricity Substations," IEEE Trans. Instrum. Meas., vol. 62, no. 10, pp. 2694-2703, Oct. 2013.

[22] UCA International Users Group. (2004, July 7). Implementation Guideline for Digital Interface to Instrument Transformers using IEC 61850-9-2. (R2-1) [Online]. Available: http://iec61850.ucaiug.org/Implementation\%20Guidelines/DigIF_spec_ 9-2LE R2-1 040707-CB.pdf

[23] F. Steinhauser, C. Riesch and M. Rudigier, "IEEE 1588 for Time Synchronization of Devices in the Electric Power Industry," in Proc. 2010 IEEE Int. Symp. Precis. Clock Synchr. Meas. Control Commun. (ISPCS), Portsmouth, USA, 27 Sep. - 01 Oct. 2010, pp. 1-6.

[24] L. Heng, T. Walter, P. Enge and G. X. Gao, "GNSS Multipath and Jamming Mitigation using High-Mask-Angle Antennas and Multiple Constellations," IEEE Trans. Intell. Transp. Syst., vol. 16, no. 2, pp. 741-750, Mar. 2015.

[25] IEEE Standard for Local and metropolitan area networks: Media Access Control (MAC) Bridges, IEEE Std 802.1D-2004, Jun. 2004.

[26] Industrial communication networks - High availability automation networks Part 3: Parallel Redundancy Protocol (PRP) and High-availability Seamless Redundancy (HSR), IEC 62439-3:2012, Sep. 2012.

[27] G. Prytz, "Network Recovery Time Measurements of RSTP in an Ethernet Ring Topology", in Proc. 2007 IEEE Conf. Emerging Technologies and Factory Automation (ETFA), Patras, Greece, 25-28 Sep. 2007, pp. 1427-1253.

[28] Chronos Technology. (2013, July). GPS Antenna Installations Best Practice. (r1.1) [Online]. Available: www.chronos.co.uk/files/pdfs/cs-an/GPS-Installation-Best-Practice.pdf

[29] PCS-221G Merging Unit Instruction Manual, 1.00 ver., NR Electric Co., Nanjing, Jiangsu, 2012, pp. 11-12.

[30] Technical Manual Current Differential Protection Relays, P544\&6/EN/TM/M, Alstom Grid, Stafford, pp.6-10.

\section{BIOGRAPHIES}

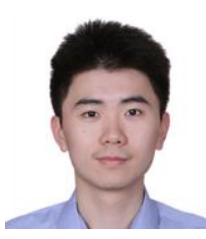

Hao Guo received the BEng. Degree from the University of Manchester, Manchester, UK in 2012. He is currently a $\mathrm{PhD}$ student at The University of Manchester. His research interests include time synchronization, Ethernet redundancy and engineering within power substations.

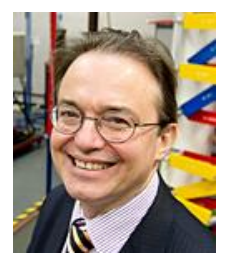

Peter Crossley received the B.Sc. degree from UMIST, Manchester, U.K., in 1977 and the Ph.D. degree from the University of Cambridge, U.K., in 1983. He is a Professor of Electrical Power Systems Engineering at The University of Manchester, U.K. and Director of Doctoral Training Centre. He has published over 250 technical papers on protection and is an active member of various CIGRE, IEEE, and IET committees. 